\title{
Numerical methods for piecewise constant Robin coefficient
}

\author{
Yanbo Ma \\ College of Mathematics and Statistics, Hanshan Normal University, Chaozhou, Guangdong, 521041, P. R. China.
}

\begin{abstract}
In this paper, we consider a numerical method for reconstructing piecewise constant Robin coefficients from boundary measurements. An adaptive total variation functional is proposed. The boundary integral equation method is utilized for discretizing the functional, and the Gauss-Newton method is employed for solving the non-linear problem. (C)2017 all rights reserved.
\end{abstract}

Keywords: Inverse problem, ill-posedness, boundary integral equations, total variation, Robin coefficient. 2010 MSC: 45Q05, 65N21.

\section{Introduction}

Let $\Omega$ be a smooth bounded domain in $R^{2}$ with boundary $\Gamma$. Consider the Robin boundary value problem for the Laplace equation

$$
\begin{cases}\Delta u=0, & \text { in } \Omega, \\ \frac{\partial u}{\partial v}+p u=g, & \text { on } \partial \Omega=\Gamma .\end{cases}
$$

Here $\vec{v}$ is the unit outward norm direction on $\Gamma . p=p(x)$ is the Robin coefficient with support contained in $\Gamma_{1} \subset \Gamma$, and $g=g(x)$ is a prescribed input function.

In this paper, we are interested in the reconstruction of an unknown piecewise constant Robin parameter $p$ from a measurement of the solution $u=u_{0}$ on another part $\Gamma_{0}$ of the boundary, where $\Gamma_{0} \cap \Gamma_{1}=\emptyset$. The inverse problem arises from various nondestructive evaluation methods, where an unknown material profile in a non-accessible part of the boundary is to be recovered from a partial boundary measurement made on an accessible part of the boundary. For example, in corrosion detection $[12,17,23,24]$, $p$ represents the corrosion damage profile on a non-accessible boundary, and $u_{0}$ is the electrostatic measurement made on an accessible boundary. In the study of MOSFET semiconductor devices [1, 7, 20, 21], the Robin coefficient $p$ contains information on the quality and location of the non-accessible metal-to-silicon contact window, and voltage measurement $\mathfrak{u}_{0}$ on an accessible part is used to extract $p$.

The main purpose of this paper is to present a numerical method for the reconstructing a piecewise constant Robin parameter in two-dimensional case.

The Robin inverse problem has been the subject of many recent studies, and most of them are concerned with important properties of the forward map from $p$ to $u_{0}$, such as uniqueness, continuity with

Email address: yanboma@hstc.edu.cn (Yanbo Ma)

doi:10.22436/jmcs.017.01.09

Received 2015-12-24 
respect to proper norms, and differentiability and stability in various forms. Some numerical methods of recovering the Robin coefficient have been proposed [3, 5, 6, 8-11, 13, 14, 16, 19]. However, these methods are not applied in the reconstruction of piecewise constant Robin coefficient.

In the case of a piecewise constant Robin coefficient, Jin and Zou [15] proposed a variational formulation based on the Modica-Mortola functional. This method has the advantage of being robust to noise, however, many parameters are naturally involved because of the regularization of the class of admissible parameters by $\mathrm{H}^{1}$ functions. Chaabane et al. [4] presented a numerical method in the two- and threedimension case based on the Kohn-Vogelius method, however, they do not consider the regularization term of Robin coefficient $p$ in the proposed cost functional.

We first recast the inverse problem as an integral equation formulation, which is particularly advantageous when we look for numerical solution for both the forward and inverse problem. However the inverse problem is still an ill-posed problem in Hadamard's sense because it is sensitive to errors no measured data. To prevent such a blowing up, we penalize the oscillatory behaviors by adding an adaptive total variation regularization term in the least square function to be minimized. We present numerical method for the Robin inverse problem based on the objective functional that consists of least squares functional and a regularization term. Gauss-Newton method is employed for the solution of the optimization problem, and we introduce the adjoint method to solve the gradient and the Hessian matrix of the nonlinear fit-to-data functional.

The rest of the paper is organized as follows. Section 2 formulates the inverse problem by boundary integral equations, and the numerical methods based on fit-to-data least squares and regularization are presented. In Section 3, we present the adaptive TV functional for the inverse problem, and we discuss discretization for planar domains, in particular for ellipses in Section 4. In Section 5 we solve the gradient and Hessian matrix of the nonlinear fit-to-data functional, and then solve the optimization problem by using the Gauss-Newton method. Numerical examples are then presented in Section 6 to show the effectiveness of our numerical methods in recovering the Robin coefficients from noisy data.

\section{Nonlinear integral equation}

We first introduce the boundary integral equation formulation for boundary value problem (1.1). We assume that the domain $\Omega$ has a smooth boundary $\Gamma$, the closed subsets $\Gamma_{0}$ and $\Gamma_{1}$ are disjoint, and $p(t)$ is a nonnegative function on $\Gamma$ with $\operatorname{supp}(p) \subset \Gamma_{1}$. Let $\Phi=\Phi(x, y)$ be the fundamental solution for the Laplacian in $\mathrm{R}^{2}$ :

$$
\Phi(x, y)=\frac{1}{2 \pi} \ln \frac{1}{|x-y|} \quad \text { for } \quad x \neq y .
$$

From now on, without loss of generality, we assume that there exists a point $x_{0} \in \Omega$ such that $\left|x-x_{0}\right| \neq 0$ for all $x \in \partial \Omega$. Then Theorem 3.16 in [2] guarantees that the corresponding single-layer boundary integral operator is injective.

As shown in [2], the PDE formulation (1.1) for $u \in H^{1}(\Omega)$ is equivalent to the following integral equation for the trace of $u$ on $\Gamma$, denote by $u \in H^{1 / 2}(\Gamma)$ :

$$
\frac{1}{2} \mathfrak{u}(x)+\int_{\Gamma}\left(\frac{\partial \Phi(x, y)}{\partial v_{y}}+p(y) \Phi(x, y)\right) \mathfrak{u}(y) d s_{y}=\int_{\Gamma} \Phi(x, y) g(y) d s_{y}, \quad x \in \Gamma .
$$

Define

$$
\begin{aligned}
& (\mathcal{D} u)(x)=\int_{\Gamma} \frac{\partial \Phi(x, y)}{\partial v_{y}} u(y) d s_{y} \\
& (\mathcal{S u})(x)=\int_{\Gamma} \Phi(x, y) u(y) d s_{y}
\end{aligned}
$$

Then (2.1) can be written as

$$
\mathcal{A}(p)(u):=\left(\frac{1}{2} \mathcal{J}+\mathcal{D}\right) \mathfrak{u}+\mathcal{S}(p \mathfrak{u})=\mathcal{S} g .
$$


The system of integral equations (2.1) and (2.2) equivalent to our inverse problem is not unique. In fact, represent the solution $u$ as surface superposition of point sources given by the fundamental solution with an unknown density one can derive a different system of integral equation equivalent to our inverse problem.

For the further investigation of the nonlinear integral equation, we introduce a parameterization on the integral equation for the numerical solution. In this preliminary study, for the sake of simplicity we confine ourselves to smooth boundaries $\Gamma$ of class $\mathcal{C}^{2}$, that is, we represent

$$
\Gamma:=\{z(t): t \in[0,2 \pi]\}
$$

with a $2 \pi$ periodic $\mathcal{C}^{2}$-smooth function $z: \mathbb{R} \rightarrow \mathbb{R}^{2}$ such that $z$ is injective on $[0,2 \pi)$ and satisfies $z^{\prime}(t) \neq 0$ for all $t$. Setting

$$
\mathrm{u}(\mathrm{t}):=\left|z^{\prime}(\mathrm{t})\right| \varphi(z(\mathrm{t}))
$$

we obtain the parameterized integral operators

$$
(\widetilde{S} u)(t)=\frac{1}{2 \pi} \int_{0}^{2 \pi} \ln \frac{1}{|z(t)-z(\tau)|} u(\tau) d \tau
$$

and

$$
(\widetilde{\mathrm{D}} \mathrm{u})(\mathrm{t})=\frac{1}{2 \pi\left|z^{\prime}(\mathrm{t})\right|} \int_{0}^{2 \pi} \frac{\left[z^{\prime}(\mathrm{t})\right]^{\perp} \cdot[z(\mathrm{t})-z(\tau)]}{|z(\mathrm{t})-z(\tau)|^{2}} \mathrm{u}(\tau) \mathrm{d} \tau,
$$

for $t \in[0,2 \pi]$. Here, we used the notation $a^{\perp}=\left(a_{2},-a_{1}\right)$ for any vector $a=\left(a_{1}, a_{2}\right)$.

For the discretization of the integral operators we note that the $2 \pi$ periodic kernel of the $\widetilde{S}$ can be decomposed in the form

$$
\ln \frac{1}{|z(t)-z(\tau)|}=-\ln \left|\sin \frac{t-\tau}{2}\right|+\ln \frac{\sin \frac{t-\tau}{2}}{|z(t)-z(\tau)|},
$$

where the second term is smooth with diagonal values

$$
\lim _{\tau \rightarrow t} \ln \frac{\sin \frac{t-\tau}{2}}{|z(t)-z(\tau)|}=-\ln 2\left|z^{\prime}(t)\right| .
$$

Hence, the well established trigonometric interpolation quadrature rules on equidistant meshes for logarithmic singularities as described in [18] are available. The $2 \pi$ periodic kernel of $\widetilde{D}$ is smooth with diagonal term

$$
\frac{\left[z^{\prime}(t)\right]^{\perp} \cdot z^{\prime \prime}(t)}{4 \pi\left|z^{\prime}(t)\right|^{3}}
$$

and therefore the trapezoidal rule can be employed.

\section{The regularization of the inverse problem}

Note that the forward problem is to solve (2.1) or (2.3) to obtain $u$ on $\Gamma$ for given $p$, and the Robin inverse problem is to find $p$ on $\Gamma_{1}$ such that the solution $u$ of (2.1) matches the given knowledge $u_{0}$ on $\Gamma_{0}$ :

$$
\left.\mathrm{u}\right|_{\Gamma_{0}}=\mathrm{u}_{0} .
$$

We denote the restriction operator from $\Gamma$ to $\Gamma_{0}$ by $\mathcal{R}_{0}$ : that is, for $u$ defined on $\Gamma, \mathcal{R}_{0} u$ is defined on $\Gamma_{0}$ with $\left(\mathcal{R}_{0} u\right)(x)=u(x)$ for $x \in \Gamma_{0}$. Then, for a given input $g$, the measurement $u_{0}$ of $u$ on $\Gamma_{0}$ can be expressed as

$$
\mathcal{R}_{0} \mathfrak{u}=\mathfrak{u}_{0} \text {. }
$$


When the data $u_{0}$ is perturbed, the solution of the inverse problem does not depend continuously on the data even if it does exist, as easily conceived from the severe ill-posedness of the inverse problem. Hence, instead of working directly with equation (2.1), we turn to a variational formulation of the problem, thereby transforming it to an optimization problem. That is, we seek a solution to this equation match to the nonlinear constrained regularized least-squares problem:

$$
\left\{\begin{array}{l}
\min _{p} \frac{1}{2}\left\|\mathcal{R}_{0} u-u_{0}\right\|_{\mathrm{L}^{2}\left(\Gamma_{0}\right)}^{2}+\frac{\alpha}{2} \operatorname{ATV}(p), \\
\text { s.t. } \mathcal{A}(p)(u)=\delta g .
\end{array}\right.
$$

Here $A T V(p)=\int_{\Gamma} \frac{\left|\frac{\partial p}{\partial n}\right|^{2}}{\sqrt{\left|\frac{\partial p}{\partial n}\right|^{2}+\varepsilon^{2}}}$ is a adaptive total variation (ATV) regularization functional, that is, in order to cut down the nondifferentiability of the Euclidean norm at the origin, we consider the approximation to total variation (TV) functional, which was first introduced by Rudin, Osher, and Fatemi (ROF) in their pioneering work [22] on edge preserving image denoising. It was designed with the explicit goal of preserving sharp discontinuities in image while removing noise and other unwanted fine scale detail. The revolutionary aspect of the ROF model is its regularization term that allows for discontinuities but at the same time disfavors oscillations. $\alpha$ is a positive regularization parameter. However, the proposed ATV appropriate the TV when $\left|\frac{\partial p}{\partial n}\right|>>\varepsilon$, and tend to Winer filtering when $\left|\frac{\partial p}{\partial n}\right|<<\varepsilon$.

Since the forward problem (2.1) is well-posed, we denote its solution $u$ by

$$
\mathrm{u}=\mathcal{A}(\mathrm{p})^{-1} \mathrm{f},
$$

where $f=S g$. Thus, the constrained least-squares problem (3.1) is reformulated as a unconstrained leastsquares problem:

$$
\min _{p} \mathcal{J}(p)=\frac{1}{2}\left\|\mathcal{R}_{0} \mathcal{A}(p)^{-1} f-u_{0}\right\|_{\mathrm{L}^{2}\left(\Gamma_{0}\right)}^{2}+\frac{\alpha}{2} \operatorname{ATV}(p)
$$

\section{Discretization for planar domains}

In this section, we discuss the discretization of the least-squares functional (3.2) for planar domains. In particular, details are given for representative domains with elliptic boundaries. For more details, we refer readers to [19].

\subsection{Parametrization of $\Gamma$}

Suppose the boundary $\Gamma$ of a planar domain $\Omega$ has a regular parametrization given by

$$
\left(x_{1}, x_{2}\right)=(\phi(t), \psi(t)) \text { for } 0 \leqslant t \leqslant L,
$$

with $(\phi(0), \psi(0))=(\phi(\mathrm{L}), \psi(\mathrm{L}))$. Assume that $\phi(\cdot)$ and $\psi(\cdot)$ are both $\mathrm{C}^{2}$ functions.

Define

$$
K_{s}(t, s)=-\frac{1}{2 \pi}\left(\ln \sqrt{(\phi(s)-\phi(t))^{2}+(\psi(s)-\psi(t))^{2}}\right) \sqrt{(\phi(s))^{2}+(\psi(s))^{2}}
$$

and

$$
K_{d}(t, s)=\frac{1}{2 \pi} \frac{\psi^{\prime}(s)(\phi(t)-\phi(s))+\phi^{\prime}(s)(\psi(t)-\psi(s))}{(\phi(s)-\phi(t))^{2}+(\psi(s)-\psi(t))^{2}}
$$

for $x=(\phi(t), \psi(t))$ and $y=(\phi(s), \psi(s))$ on $\Gamma$ with $x \neq y(t \neq s)$. We can write the operators $\mathcal{S}$ and $\mathcal{D}$ as

$$
\begin{aligned}
& (\mathcal{S u})(\mathrm{t})=\int_{0}^{\mathrm{L}} \mathrm{K}_{\mathrm{s}}(\mathrm{t}, \mathrm{s}) \mathrm{u}(\mathrm{s}) \mathrm{ds}, \\
& (\mathcal{D u})(\mathrm{t})=\int_{0}^{\mathrm{L}} \mathrm{K}_{\mathrm{d}}(\mathrm{t}, \mathrm{s}) \mathrm{u}(\mathrm{s}) \mathrm{ds},
\end{aligned}
$$

where $u(s)=u(\phi(s), \psi(s))$. 


\subsection{Discretization}

We use the mid-point quadrature rule to discretize the integral operators and central difference quotients to approximate derivatives. Let the interval $[0, \mathrm{~L}]$ be partitioned into $\mathrm{n}$ uniform subintervals: $[(i-1) h, i h], i=1,2, \ldots, n$, where $h=L / n$. Then the quadrature points are $t_{i}=(i-1 / 2) h, i=1,2, \ldots, n$. Suppose

$$
\left\{t_{i}\right\}_{i=1}^{n} \bigcap\left\{t:(\phi(t), \psi(t)) \in \Gamma_{0}\right\}=\left\{t_{m_{1}+1}, \ldots, t_{m_{2}}\right\}
$$

and

$$
\left\{\mathrm{t}_{\mathrm{i}}\right\}_{i=1}^{n} \bigcap\left\{\mathrm{t}:(\phi(\mathrm{t}), \psi(\mathrm{t})) \in \Gamma_{1}\right\}=\left\{\mathrm{t}_{\mathrm{m}_{3}+1}, \ldots, \mathrm{t}_{\mathrm{m}_{4}}\right\} .
$$

Denote by $\mathbf{u}$ and $\mathbf{p}$ the discretized functions of $u(t)$ on $\Gamma$, and $p(t)$ on $\Gamma_{1}$, respectively:

$$
\mathbf{u}=\left[u\left(t_{1}\right), \ldots, u\left(t_{n}\right)\right]^{\top}, \quad \mathbf{p}=\left[p\left(t_{m_{3}+1}\right), \ldots, p\left(t_{m_{4}}\right)\right]^{\top}
$$

and the discrete data

$$
\mathbf{u}_{0}=\left[\mathrm{u}_{0}\left(\mathrm{t}_{\mathrm{m}_{1}+1}\right), \ldots, \mathrm{u}_{0}\left(\mathrm{t}_{\mathrm{m}_{2}}\right)\right]^{\top}, \quad \mathbf{f}=\left[\mathrm{f}\left(\mathrm{t}_{1}\right), \ldots, f\left(\mathrm{t}_{\mathrm{n}}\right)\right]^{\top} .
$$

Let the matrix representation of the kernel $\mathrm{K}_{\mathrm{d}}$ be denoted by $\mathrm{D}$ and that of $\mathrm{K}_{\mathrm{s}}$ be denoted by $\mathrm{S}$. Then the matrix representation of $\mathcal{A}(p)$ in (2.3) is

$$
A(\mathbf{p})=\frac{1}{2} \mathrm{I}+\mathrm{D}+\mathrm{SP},
$$

where $I$ is the identity matrix of order $n$ and $P=\operatorname{diag}(\mathbf{p})$ with

$$
p_{i}= \begin{cases}p\left(t_{i}\right), & i=m_{3}+1, \ldots, m_{4} \\ 0, & \text { others. }\end{cases}
$$

As for the one dimension regularization term, we get the discretion of the ATV:

$$
\operatorname{ATV}_{\varepsilon}(\mathbf{p})=h \sum_{i=m_{3}+1}^{m_{4}+1} \frac{\left|\mathbf{p}_{i}-\mathbf{p}_{i-1}\right|^{2}}{\sqrt{\left|\mathbf{p}_{i}-\mathbf{p}_{i-1}\right|^{2}+\varepsilon^{2}}},
$$

where $\varepsilon$ is a small positive parameter, and $p_{m_{3}}=0$, and $p_{m_{4}+1}=0$. We note that since

$$
\left(\phi\left(t_{m_{3}}\right), \psi\left(t_{m_{3}}\right)\right),\left(\phi\left(t_{m_{4}+1}\right), \psi\left(t_{m_{4}+1}\right)\right) \notin \Gamma_{1},
$$

$p_{m_{3}}=p\left(t_{m_{3}}\right)=0, p_{m_{4}+1}=p\left(t_{m_{4}+1}\right)=0$.

The least-square functional (3.2) then becomes

$$
\min _{\mathbf{p}}\left\{\frac{1}{2}\left\|\mathrm{R}_{0} \mathrm{~A}(\mathbf{p})^{-1} \mathbf{f}-\mathbf{u}_{0}\right\|^{2}+\alpha \mathrm{ATV}(\mathbf{p})\right\}
$$

where $R_{0}$ is the discretization of the restriction operator $\mathcal{R}_{0}$, i.e.,



Here $\mathrm{O}_{\mathrm{r} \times \mathrm{s}}$ denotes the $\mathrm{r} \times \mathrm{s}$ zero matrix and $\mathrm{I}_{\mathrm{r}}$ denotes the identity matrix of order $\mathrm{r}$.

\subsection{Elliptic domains}

Suppose $\Omega$ is an ellipse in $R^{2}$ :

$$
\Omega=\left\{\left(x_{1}, x_{2}\right):\left(\frac{x_{1}}{a}\right)^{2}+\left(\frac{x_{2}}{b}\right)^{2}<1\right\}
$$

with $a, b>0$. The usual parametrization for $\Gamma$ is

$$
\left(x_{1}, x_{2}\right)=(\phi(t), \psi(t))=(a \cos (2 \pi t), b \sin (2 \pi t)) \text { for } 0 \leqslant t \leqslant 1 .
$$

In this case, the kernels in the integral operators can explicitly be expressed as 


$$
\begin{aligned}
& K_{s}(t, s)=-\ln \left(2|\sin (\pi(t-s))| \sqrt{a^{2} \sin ^{2}(\pi(t+s))+b^{2} \cos ^{2}(\pi(t+s))}\right) \times \sqrt{a^{2} \sin ^{2}(2 \pi(s))+b^{2} \cos ^{2}(2 \pi(s))} \\
& K_{d}(t, s)=-\frac{a b}{2\left(a^{2} \sin ^{2}(\pi(t+s))+b^{2} \cos ^{2}(\pi(t+s))\right)}
\end{aligned}
$$

for $0 \leqslant t, s \leqslant 1$.

Since $K_{d}(t, s)$ is smooth, we have

$$
\mathrm{D}=\mathrm{h}\left[\mathrm{K}_{\mathrm{d}}\left(\mathrm{t}_{\mathrm{i}}, \mathrm{t}_{j}\right)\right]_{i, j=1}^{\mathrm{n}} \text {. }
$$

As for $K_{s}(t, s)$, since it is weakly singular at $s=t$ and $(t, s)=(0,1)$ and $(1,0)$, special case should be taken in discretizing $\int_{0}^{1} K_{s}(t, s) u(s) d s$ to avoid large errors, see [19]. By decomposing $K_{s}(t, s)$ as

$$
K_{s}(t, s)=\left(K_{s 1}(t, s)+K_{s 2}(t, s)\right) K_{s 3}(t, s),
$$

where

$$
\begin{aligned}
K_{s 1}(t, s) & =\ln (2|\sin (\pi(t-s))|), \\
K_{s 2}(t, s) & =\ln \left(\sqrt{a^{2} \sin ^{2}(\pi(t+s))+b^{2} \cos ^{2}(\pi(t+s))}\right), \\
K_{s 3}(s) & =-\sqrt{a^{2} \sin ^{2}(2 \pi(s))+b^{2} \cos ^{2}(2 \pi(s))},
\end{aligned}
$$

and using the fact that

$$
\int_{0}^{1} \ln |2 \sin (\pi s)| d s=0,
$$

one can remove the singularity by subtraction:

$$
\int_{0}^{1} K_{s}(t, s) u(s) d s=\int_{0}^{1} K_{s 1}(t, s)\left[K_{s 3}(s) u(s)-K_{s 3}(t) u(t)\right] d s+\int_{0}^{1} K_{s 2}(t, s) K_{s}(s) u(s) d s .
$$

It follows that

$$
\int_{0}^{1} K_{s}\left(t_{i}, s\right) u(s) d s \approx h \sum_{j=1}^{n}\left(K_{s}\left(t_{i}, t_{j}\right)-\delta_{i j} \sum_{k=1}^{n} K_{s 1}\left(t_{i}, t_{k}\right) K_{s 3}\left(t_{i}\right)\right) u\left(t_{j}\right),
$$

where $\delta_{i j}$ is the Kronecker symbol, i.e., $\delta_{i j}=1$ if $i=j$ and 0 otherwise. Therefore,

$$
S=\left[h K_{s}\left(t_{i}, t_{j}\right)-\delta_{i j} h \sum_{k=1}^{n} K_{s 1}\left(t_{i}, t_{k}\right) K_{s 3}\left(t_{i}\right) u\left(t_{i}\right)\right]_{i, j=1}^{n} .
$$

\section{The Gauss-Newton method}

We consider solving the regularized minimization problem (4.1) by using the well-known GaussNewton method (GNM), which is one of the most popular quasi-Newton methods for solving nonlinear optimization problems without constraints. This method has been proven to have good performance even for non-smooth optimizations.

We see that in order to solve (4.1) by using the Gauss-Newton algorithm, we are required to compute the gradients and the Hessian matrix of $F(\mathbf{p}) \equiv \frac{1}{2}\left\|R_{0} A(\mathbf{p})^{-1} \mathbf{f}-\mathbf{u}_{0}\right\|_{2}^{2}$ and $\operatorname{TV}(\mathbf{p})$. We note that

$$
\begin{aligned}
\frac{\partial}{\partial p_{i}} R_{0} A(\mathbf{p})^{-1} \mathbf{f} & \left.=\lim _{\tau \rightarrow 0} R_{0}\left[A\left(\mathbf{p}+\tau \mathbf{e}_{i}\right)^{-1}-A(\mathbf{p})^{-1}\right)\right] \mathbf{f} / \tau \\
& =\lim _{\tau \rightarrow 0} R_{0} A\left(\mathbf{p}+\tau \mathbf{e}_{i}\right)^{-1} \cdot\left[A(\mathbf{p})-A\left(\mathbf{p}+\tau \mathbf{e}_{i}\right)\right] / \tau \cdot A(\mathbf{p})^{-1} \mathbf{f} \\
& =-R_{0} A(\mathbf{p})^{-1} S \operatorname{diag}\left(\delta_{m_{3}+i, 1}, \ldots, \delta_{m_{3}+i, n}\right) A(\mathbf{p})^{-1} \mathbf{f},
\end{aligned}
$$


where $\mathbf{e}_{i}$ is the $i$ th column of the identity matrix of order $n$. Denoting $A(\mathbf{p})^{-1} \mathbf{f}$ by $\mathbf{u}_{\mathbf{p}}=\left(u_{1}, \ldots, u_{n}\right)^{\top}$, then

It follows that

$$
\frac{\partial}{\partial p_{i}} R_{0} A(\mathbf{p})^{-1} \mathbf{f}=-R_{0} A(\mathbf{p})^{-1} S\left(0, \ldots, 0, u_{m_{3}+i}, 0, \ldots, 0\right)^{\top} .
$$

Thus, we get

$$
\frac{\partial}{\partial p_{i}} F(\mathbf{p})=-\left(0, \ldots, 0, u_{m_{3}+i}, 0, \ldots, 0\right) S^{\top} A(\mathbf{p})^{-\top} R_{0}^{\top}\left(R_{0} \mathbf{u}_{p}-\mathbf{u}_{0}\right) .
$$

$$
\nabla F(\mathbf{p})=-\operatorname{diag}\left(u_{m_{3}+1}, \ldots, u_{m_{4}}\right) S_{1}^{\top} A(\mathbf{p})^{-\top} R_{0}^{\top}\left(R_{0} \mathbf{u}_{\mathbf{p}}-\mathbf{u}_{0}\right),
$$

where $S_{1}=S\left(\cdot, m_{3}+1: m_{4}\right)$.

The Hessian of the $\mathcal{F}(p)$ can be expressed as

$$
\mathrm{H}(\mathbf{p})=\mathrm{H}^{\mathrm{GN}}(\mathbf{p})+\frac{\mathrm{d}^{2} \mathcal{R}, \mathrm{A}(\mathbf{p})^{-1} \mathbf{f}}{\mathrm{d} \mathbf{p}^{2}}(\mathcal{R}, \mathrm{A}(\mathbf{p}) \mathrm{u}-\mathbf{f}),
$$

where

$$
\begin{aligned}
H^{\mathrm{GN}}(\mathbf{p}) & =\left(\frac{\mathrm{d} \mathcal{R}, \mathrm{A}(\mathbf{p})^{-1} \mathbf{f}}{\mathrm{d} \mathbf{p}}\right)^{*} \frac{\mathrm{d} \mathcal{R}, \mathrm{A}(\mathbf{p})^{-1} \mathbf{f}}{\mathrm{d} \mathbf{p}} \\
& =\left(\mathrm{R}_{0} \mathrm{~A}(\mathbf{p})^{-1} \mathrm{~S}_{1} \operatorname{diag}\left(\mathrm{u}_{\mathrm{m}_{3}+1}, \ldots, \mathrm{u}_{\mathrm{m}_{4}}\right)\right)^{\mathrm{T}}\left(\mathrm{R}_{0} \mathrm{~A}(\mathbf{p})^{-1} \mathrm{~S}_{1} \operatorname{diag}\left(\mathrm{u}_{\mathrm{m}_{3}+1}, \ldots, \mathrm{u}_{\mathrm{m}_{4}}\right)\right)
\end{aligned}
$$

is the Gauss-Newton approximation to the Hessian. This has several computational advantages. First, it can be much easier to compute than the full Hessian, since it does not involve the second derivative term. Second, the Gauss-Newton approximation is positive semidefinite, and it is positive definite if the first derivative has full rank. This guarantees that the Gauss-Newton step is a descent direction.

It is easy to see that the gradient of $\operatorname{ATV}_{\mathcal{E}}(\mathbf{p})$ is given by

$$
\nabla \operatorname{ATV}_{\varepsilon}(\mathbf{p})=E^{\top} \operatorname{diag}(\phi(\mathbf{p})) E \mathbf{p},
$$

where $\operatorname{diag}(\phi(\mathbf{p}))$ denotes the diagonal matrix whose ith diagonal entry is $\frac{\left(\left(E_{i} \mathbf{p}\right)^{2}+2 \beta^{2}\right)}{\left(\left(E_{i} \mathbf{p}\right)^{2}+\beta^{2}\right)^{\frac{3}{2}}}$, and the Hessian of $\operatorname{TV}_{\mathcal{E}}(\mathbf{p})$ is given by

$$
\operatorname{Hess}\left(A \operatorname{TV}_{\varepsilon}(\mathbf{p})\right)=E^{\top} \operatorname{diag}(\psi(\mathbf{p})) E,
$$

where $\operatorname{diag}(\psi(\mathbf{p}))$ denotes diagonal matrix whose ith diagonal entry is

$$
\frac{\left[\left(E_{i} \mathbf{p}\right)^{2}+\varepsilon^{2}\right]\left[3\left(E_{i} \mathbf{p}\right)^{2}+2 \varepsilon^{2}\right]-2\left[\left(E_{i} \mathbf{p}\right)^{2}+2 \varepsilon^{2}\right]\left(E_{i} \mathbf{p}\right)^{2}}{\left(\left(E_{i} \mathbf{p}\right)^{2}+\varepsilon^{2}\right)^{\frac{5}{2}}}=\frac{\left(E_{i} \mathbf{p}\right)^{2}+\varepsilon\left(E_{i} \mathbf{p}\right)+2 \varepsilon^{2}}{\left(\left(E_{i} \mathbf{p}\right)^{2}+\varepsilon^{2}\right)^{\frac{5}{2}}}
$$

and

$$
E=\left(\begin{array}{ccccc}
-1 & 1 & & & \\
0 & -1 & 1 & & \\
& \ddots & \ddots & \ddots & \\
& & & -1 & 1 \\
& & & 0 & -1
\end{array}\right) .
$$

Since an expression for the gradient and Hessian of the object functional is explicitly available, the Gauss-Newton iteration can be readily applied to the optimization problem.

\section{Numerical examples}

In this section, we use the Gauss-Newton method to solve the inverse problem on elliptic domains.

Our tests are carried out by using Matlab. We set uniform partitions on the parameter domain $[0, \mathrm{~L}]$ with $h=0.0025$. In all the examples below, we first choose $p(t)$ and $g(t)$ and obtain the solution $u(t)$ by solving (2.3), and produce the data $\mathfrak{u}_{0}$ from $\left.u\right|_{\Gamma_{0}}$ with certain levels of random noise added:

$$
u_{0}\left(t_{i}\right)=u\left(t_{i}\right)+\delta * \operatorname{rand}(m 2-m 1,1), \quad i=m_{1}+1, \ldots, m_{2},
$$


where the symbol 'rand' denotes a random number from the uniform distribution of interval $(0,1)$, and $\delta$ is the absolute noise level.

Consider the problem with the following parameters:

$$
\begin{aligned}
& a=1, b=0.2, \\
& \Gamma_{0}=\{(a \cos (2 \pi t), b \sin (2 \pi t)): t \in[0.65,0.85]\} \quad \text { (a centred segemet on the bottom half), } \\
& \Gamma_{1}=\{(a \cos (2 \pi t), b \sin (2 \pi t)): t \in[0.15,0.45]\} \quad \text { (a centred segemet on the top half), } \\
& g(a \cos (2 \pi t)), b \sin (2 \pi t))= \begin{cases}1, & \text { if } t \in[0.4,0.6], \\
0, & \text { elsewhere on } \Gamma .\end{cases}
\end{aligned}
$$

In our experiments, we consider the different cases about the unknown Robin parameter $\mathbf{p}_{\text {true }}$ : the first one deals with two singularity point, and in the second numerical test, the unknown parameter $\mathbf{p}_{\text {true }}$ presents four singularity points. Figure 1 illustrates the convergence of the forward algorithm. The $\mathrm{L}^{1}$ relative error between the numerical solution $\mathbf{p}_{\mathrm{num}}$ and $\mathbf{p}_{\text {true }}$ is equal to $10^{-3}$ with 30 iterations in the case of two singular points and to $10^{-4}$ with 40 iterations for the second example (see Figure 2).
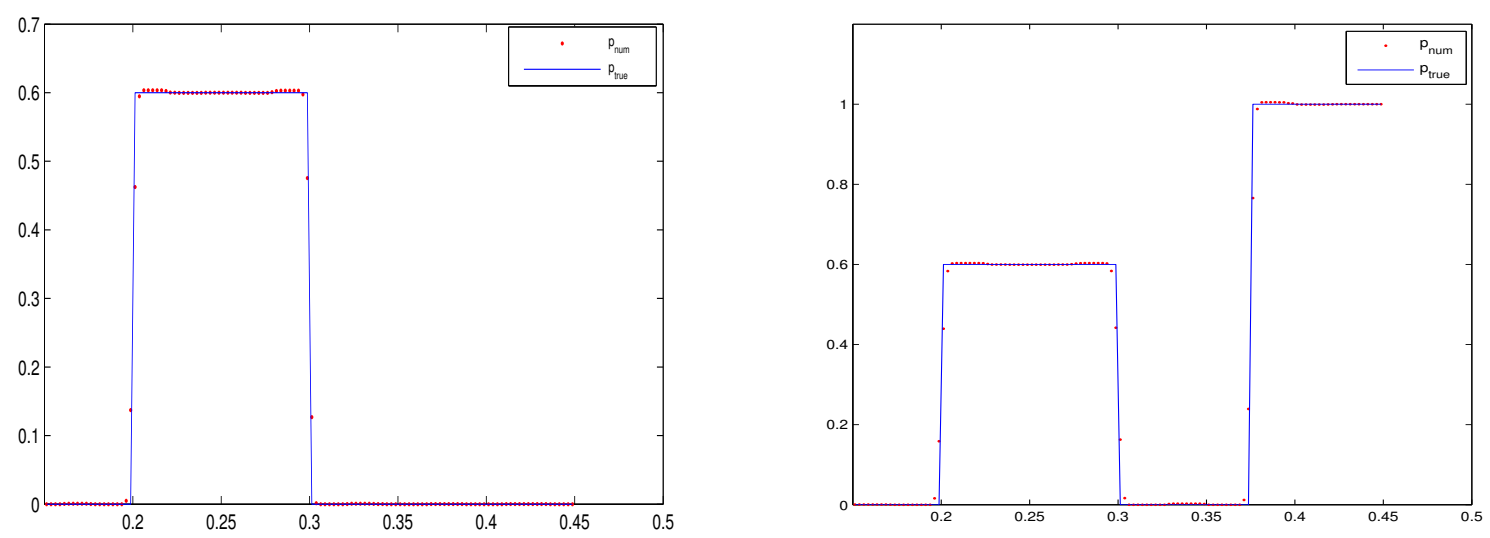

Figure 1: Reconstructions of a singular Robin parameter. (left) The case of two singular points. (right) The case of tree singular points.
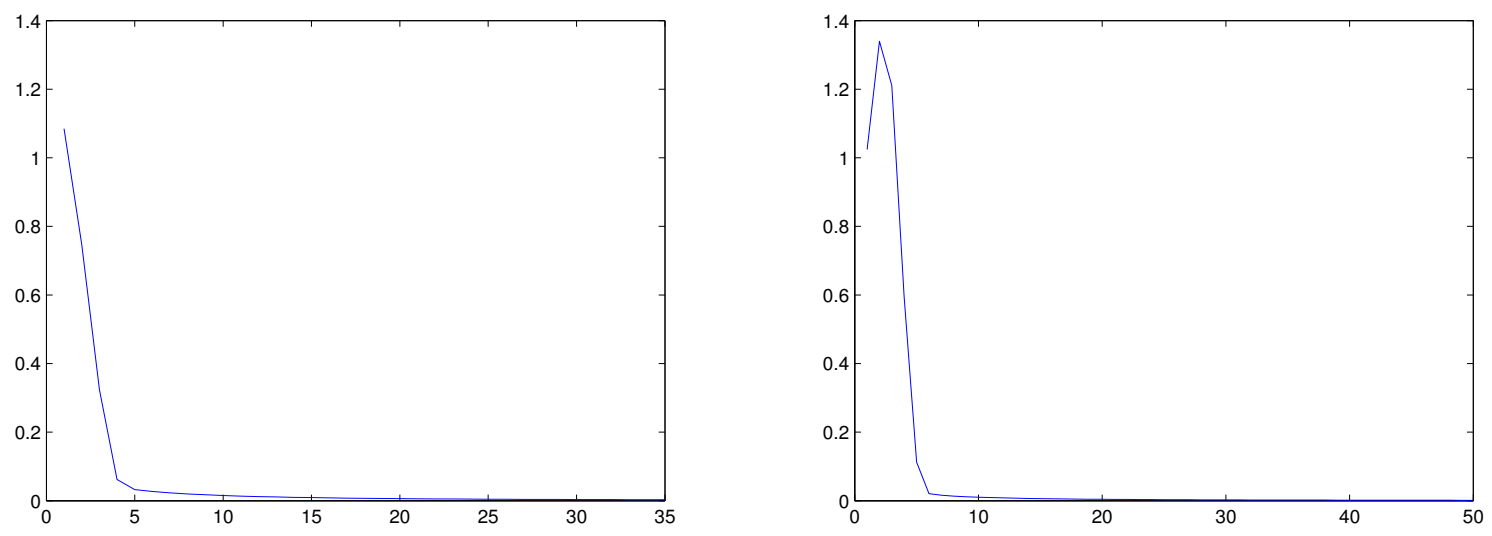

Figure 2: The $\mathrm{L}^{1}$ relative error between $p_{\text {true }}$ and $p_{\text {num }}$ with respect to number of iteration. (left) The case of two singular points. (right) The case of tree singular points. 
In the following figures, we present some numerical tests with different values of noisy data.

For $5 \%$ of noisy data (see Figure 3 (a), (b)), we have an error of $1.3 \%$ after 55 iterations for two singular point and $1.6 \%$ after 70 iterations for four singular points.

For $10 \%$ of noisy data (see Figure 3 (c), (d)), we have an error of $2.1 \%$ after 86 iterations for two singular point and 3\% after 90 iterations for four singular points.

For $15 \%$ of noisy data (see Figure 3 (e), (f)), we have an error of $4.3 \%$ after 100 iterations for two singular point and $5.2 \%$ after 110 iterations for four singular points.

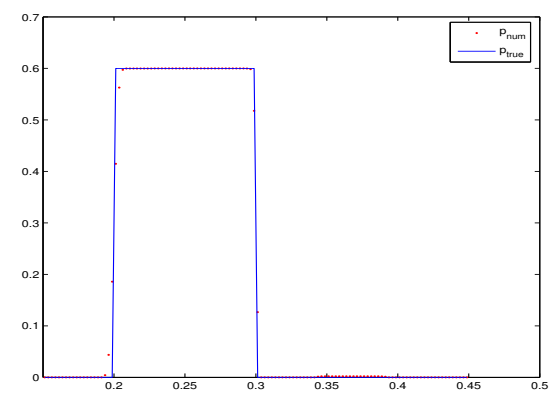

(a)

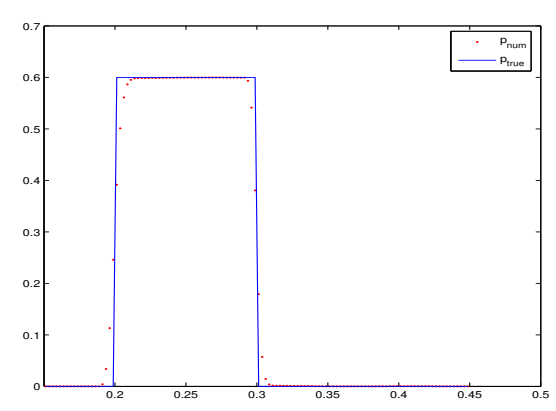

(c)

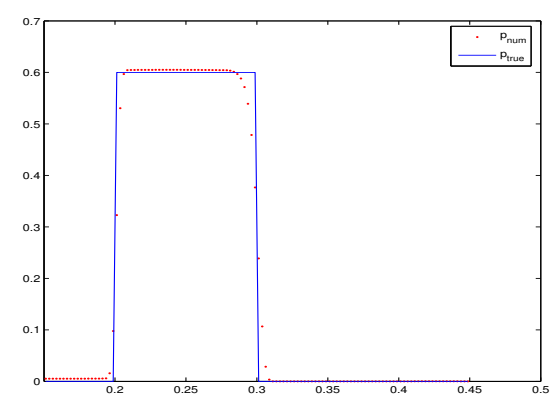

(e)

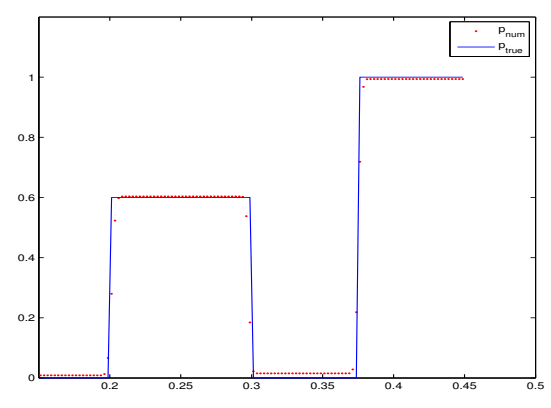

(b)

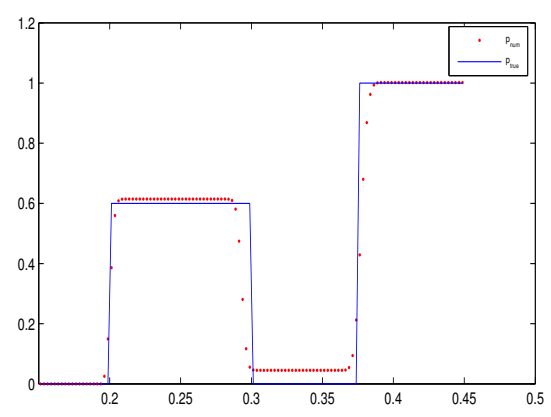

(d)

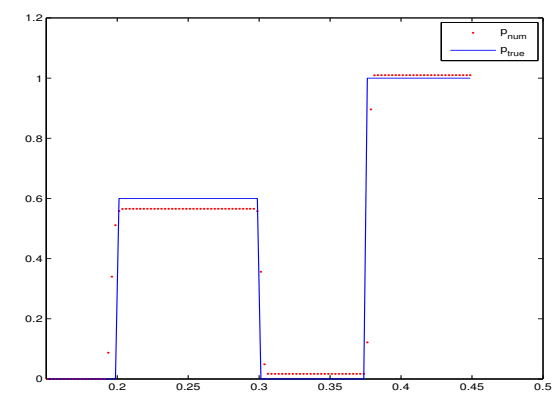

(f)

Figure 3: Reconstruction of $p_{\text {true }}$ with noisy data.

\section{Conclusion}

Because of its severe ill-posedness, it is difficult to design accurate and robust reconstruction methods for the Robin inverse problem. We have formulated the problem in the setting of boundary integral equations, and presented a numerical algorithm of gradient type solving the Robin inverse problem in 
the class of piecewise constant functions in two dimensional case. This way of solving the inverse problem has the advantage of being accurate, robust and much faster than other methods.

\section{References}

[1] S. Busenberg, W.-F. Fang, Identification of semiconductor contact resistivity, Quart. Appl. Math., 49 (1991), 639-649. 1

[2] F. Cakoni, R. Kress, Integral equations for inverse problems in corrosion detection from partial Cauchy data, Inverse Probl. Imaging, 1 (2007), 229-245. 2

[3] S. Chaabane, C. Elhechmi, M. Jaoua, A stable recovery method for the Robin inverse problem, Math. Comput. Simulation, 66 (2004), 367-383. 1

[4] S. Chaabane, I. Feki, N. Mars, Numerical reconstruction of a piecewise constant Robin parameter in the two- or threedimensional case, Inverse Problems, 28 (2012), 19 pages. 1

[5] S. Chaabane, M. Jaoua, Identification of Robin coefficients by the means of boundary measurements, Inverse Problems, 15 (1999), 1425-1438. 1

[6] S. Chaabane, M. Jaoua, J. Leblond, Parameter identification for Laplace equation and approximation in Hardy classes, J. Inverse Ill-Posed Probl., 11 (2003), 33-57. 1

[7] W.-F. Fang, F. Cumberbatch, Inverse problems for metal oxide semiconductor field-effect transistor contact resistivity, SIAM J. Appl. Math., 52 (1992), 699-709. 1

[8] W.-F. Fang, M.-Y. Lu, A fast collocation method for an inverse boundary value problem, Internat. J. Numer. Methods Engrg., 59 (2004), 1563-1585. 1

[9] W.-F. Fang, S.-X. Zeng, A direct solution of the Robin inverse problem, J. Integral Equations Appl., 21 (2009), $545-557$.

[10] W.-F. Fang, S.-X. Zeng, Numerical recovery of Robin boundary from boundary measurements for the Laplace equation, J. Comput. Appl. Math., 224 (2009), 573-580.

[11] D. Fasino, G. Inglese, An inverse Robin problem for Laplace's equation: theoretical results and numerical methods, Conference on Inverse Problems, Control and Shape Optimization, Carthage, (1998), Inverse Problems, 15 (1999), 41-48. 1

[12] G. Inglese, An inverse problem in corrosion detection, Inverse Problems, 13 (1997), 977-994. 1

[13] B.-T. Jin, Conjugate gradient method for the Robin inverse problem associated with the Laplace equation, Internat. J. Numer. Methods Engrg., 71 (2007), 433-453 1

[14] B.-T. Jin, J. Zou, Inversion of Robin coefficient by a spectral stochastic finite element approach, J. Comput. Phys., 227 (2008), 3282-3306. 1

[15] B.-T. Jin, J. Zou, Numerical estimation of piecewise constant Robin coefficient, SIAM J. Control Optim., 48 (2009), 1997-2002. 1

[16] B.-T. Jin, J. Zou, Numerical estimation of the Robin coefficient in a stationary diffusion equation, IMA J. Numer. Anal., 30 (2010), 677-701. 1

[17] P. G. Kaup, F. Santosa, Nondestructive evaluation of corrosion damage using electrostatic measurements, J. Nondestruct. Eval., 14 (1995), 127-136. 1

[18] R. Kress, Linear integral equations, Second edition, Applied Mathematical Sciences, Springer-Verlag, New York, (1999). 2

[19] F.-R. Lin, W.-F. Fang, A linear integral equation approach to the Robin inverse problem, Inverse Problems, 21 (2005), 1757-1772. 1, 4, 4.3

[20] W. M. Loh, K. Saraswat, R. W. Dutton, Analysis and scaling of Kelvin resistors for extraction of specific contact resistivity, IEEE Electron Dev. Lett., 6 (1985), 105-108. 1

[21] W. M. Loh, S. E. Swirhun, T. A. Schreyer, R. M. Swanson, K. C. Saraswat, Modeling and measurement of contact resistances, IEEE Trans. Electron Dev., 34 (1987), 512-524. 1

[22] L. I. Rodin, S. Osher, E. Fatemi, Nonlinear total variation based noise removal algorithms, Experimental mathematics: computational issues in nonlinear science, Los Alamos, NM, (1991), Phys. D, 60 (1992), 259-268. 3

[23] F. Santosa, M. Vogelius, J.-M. Xu, An effective nonlinear boundary condition for a corroding surface, Identification of the damage based on steady state electric data, Z. Angew. Math. Phys., 49 (1998), 656-679. 1

[24] M. Vogelius, J.-M. Xu, A nonlinear elliptic boundary value problem related to corrosion modeling, Quart. Appl. Math., 56 (1998), 479-505. 1 